\title{
The Reasons Suggesting a Close Link between Thermodynamics and Relativity
}

\author{
Jean-Louis Tane \\ Formerly with the Department of Geology, University Joseph Fourier, Grenoble, France \\ Email: tanejl@aol.com
}

How to cite this paper: Tane, J.-L. (2017) The Reasons Suggesting a Close Link between Thermodynamics and Relativity. Journal of Applied Mathematics and Physics, 5, 1710-1719. https://doi.org/10.4236/jamp.2017.59144

Received: August 4, 2017

Accepted: September 15, 2017

Published: September 18, 2017

Copyright $\odot 2017$ by author and Scientific Research Publishing Inc. This work is licensed under the Creative Commons Attribution International License (CC BY 4.0).

http://creativecommons.org/licenses/by/4.0/ (c) (i) Open Access

\begin{abstract}
Since the advent of relativity, it is widely accepted that the law of conservation of energy must include the energy created by disintegration of matter, or converted into matter. The aim of the present paper deals with the insertion of this concept into the basic equations of thermodynamics.
\end{abstract}

\section{Keywords}

Thermodynamics, Relativity, Energy, Entropy, Einstein's Mass-Energy Relation, Gravitation

\section{Introduction}

The first evocation of a link between thermodynamics and relativity seems to have been advanced by R.C. Tolman in 1928 [1]. In view of a photo now presented in Wikipedia [2] it can be imagined that he had the possibility to discuss directly this question with A. Einstein.

In the recent years, the same general topic has been treated by several authors. Some of them are mentioned in the section References [3]-[8]. The reading of their papers often requires a solid background in theoretical physics. Contrasting with this situation, the explanations presented below can be easily understood by scientists having a basic knowledge in classical thermodynamics. We will begin the discussion with the process of gas expansion into vacuum and the observation that its classical interpretation raises a problem.

\section{The Process of Gas Expansion into Vacuum and the Problem of Its Interpretation}

Let us consider an isolated system having the form of a horizontal cylinder divided into two parts separated by a mobile frictionless piston. We suppose that 
one part contains a gas noted 1, while the other part has been evacuated and the vacuum is noted 2 .

If the piston, previously locked (initial state), is liberated we know that it will move until the gas fills the whole system (final state).

In the usual interpretation of the process, the adopted reasoning can be summarized as follows. The system being isolated, its internal energy, noted $U$, is considered as constant, implying the relation:

$$
d U_{\text {syst }}=0
$$

Inside the system, if we apply to the gas the work equation:

$$
d W=-P_{e} d V
$$

we are led to write:

$$
d W_{g a s}=-P_{2} d V_{g a s}
$$

Having $P_{2}=0$ (since $P_{2}$ is the pressure of the vacuum), the obtained result is:

$$
d W_{\text {gas }}=0
$$

As concerns the change in heat, designated $d Q$, the usual proposal is:

$$
d Q_{\text {gas }}=0
$$

because we don't conceive that the gas can exchange heat with the vacuum.

If we suppose that, inside the system, the possible exchanges of energy are limited to work and heat, the equation corresponding to the change in internal energy takes the form:

$$
d U=d Q+d W
$$

One of the postulates implicitly admitted in thermodynamics is that the change in internal energy of a system passing from an initial state A to a final state $B$ is independent of the level of irreversibility of the process. Knowing that the conditions of reversibility are characterized by the relations:

$$
d Q=T_{i} d S
$$

(where $T_{i}$ is the internal temperature and $d S$ the change in entropy)

$$
d W=-P_{i} d V
$$

(where $P_{i}$ is the internal pressure and $d V$ the change in volume)

this is a way to say that, even in conditions of high irreversibility, the sum $d Q+$ $d W$ is supposed to have the same value as the sum $T_{i} d S-P_{i} d V$, itself often written $T d S-P d V$.

The consequence is that, for a determined process, the term $d U$ in Equation (6) is interpreted as being always equal to the term $d U$ in equation:

$$
d U=T_{i} d S-P_{i} d V
$$

itself often written:

$$
d U=T d S-P d V
$$

Referring to the gas evoked above, and taking into account the information 
given by Equation (4) and Equation (5), this is a way to say that we can write successively:

$$
\begin{gathered}
d U_{g a s}=d Q_{g a s}+d W_{g a s}=0 \\
d U_{g a s}=T_{g a s} d S_{g a s}-P_{g a s} d V_{g a s}=0
\end{gathered}
$$

Therefore the change in entropy of the gas is given by the relation:

$$
d S_{\text {gas }}=\frac{P_{\text {gas }} d V_{\text {gas }}}{T_{\text {gas }}}
$$

Remembering that $T$ is in Kelvin (absolute temperature), all the terms of Equation (13) appear to be positive, with the consequence that the term $d S_{\text {gas }}$ is positive too.

At this stage of the discussion, the important point to keep in mind is that the change in entropy of the gas is positive (Equation (13)) while its internal energy is considered constant (Equation (11)), as well as the internal energy of the whole system (Equation (1)). The same can be said for the vacuum, knowing that it represents the difference between the whole system and the gas.

Although this interpretation is classically admitted, it raises a problem that can be introduced as follows. Let us imagine that instead of being a vacuum, the second part of the system contains a gas whose initial pressure is $P_{2}$. Applying Equation (2) to both parts, we get:

$$
d W_{1}=-P_{2} d V_{1}, d W_{2}=-P_{1} d V_{2}
$$

Having $d V_{2}=-d V_{1}$, the second equation can be written $d W_{2}=P_{1} d V_{1}$ and the result obtained for the system is:

$$
d W_{s y s t}=d W_{1}+d W_{2}=d V_{1}\left(P_{1}-P_{2}\right)
$$

The term $d V_{1}$ being positive when $P_{1}>P_{2}$ and negative when $P_{1}<P_{2}$, the product $d V_{1}\left(P_{1}-P_{2}\right)$ is always positive. Consequently, the term $d W_{s y s t}$ always obeys the condition:

$$
d W_{\text {syst }}>0
$$

The conclusion of this observation is that when an isolated system is composed of two parts having a mutual exchange of work, its mechanical energy $W$ increases, inducing an increase in its internal energy $U$. In such conditions, the ability of having the relation $d U_{\text {syst }}=0$, suggested by Equation (1), requires that inside the system another kind of energy, namely its thermal energy $Q$, goes decreasing. The expected information is therefore an argumentation showing that the change in heat of the system obeys the relation:

$$
d Q_{s y s t}<0
$$

and more precisely:

$$
d Q_{s y s t}=-d W_{s y s t}
$$

Before seeing if it is possible, it can be noted through Equation (15) that, for a given value of $P_{1}$, the product $d V_{1}\left(P_{1}-P_{2}\right)$ and consequently the term $d W_{\text {syst }}$ - 
is all the greater as the value of $P_{2}$ is small. The highest value of $d W_{s y s t}$ is reached when $P_{2}=0$, that is when the second part of the system is a vacuum, a situation corresponding to the case initially considered. Admitting that there is no exchange of heat between the gas and the vacuum (as suggested by the proposal $d Q_{\text {gas }}=0$ given by Equation (5)), the internal energy of the system appears to be entirely conditioned by the relation $d W_{s y s t}>0$ and therefore leads to the conclusion:

$$
d U_{s y s}>0
$$

Obviously, we are confronted to a problem because this result does not agree with the postulate $d U_{s y s t}=0$ (Equation (1)) classically admitted for an isolated system.

As will be seen below, we are confronted to the same kind of problem when we consider the process of a heat exchange between two parts of an isolated system.

\section{The Process of Exchange of Heat Between Two Parts of an Isolated System}

Let us consider an isolated system divided into two parts separated by a fixed diathermic wall. In the initial state, we suppose that part 1 contains 1 liter of water at $T_{i}=10^{\circ} \mathrm{C}(283 \mathrm{~K})$, while part 2 contains 3 liters of water at $T_{i}=50^{\circ} \mathrm{C}(323$ $\mathrm{K})$. We know that the natural evolution of this system will consist in an exchange of heat between both parts, until they reach the same final temperature $T_{f}$

The classical intepretation of the process can be summarized as follows.

The system being isolated, its internal energy is considered as constant, proposal which obeys the relation $d U_{\text {syst }}=0$, numbered above as Equation (1).

Remembering from Equation (6) that the general expression of $\mathrm{d} U_{s y s t}$ is $d U_{s y s t}=d Q_{s y s t}+d W_{s y s t}$, and that, in the present case, the term $d W_{s y s t}$ is negligible or nil (reason explaining that it is often not evoked in thermodynamic textbooks) the idea is implicitly admitted that:

$$
d U_{s y s t}=d Q_{s y s t}
$$

In such conditions, combining Equation (1) $\left(d U_{s y s t}=0\right)$ and 20, leads to the relation:

$$
d Q_{s y s t}=0
$$

which implies itself:

$$
d Q_{2}=-d Q_{1}
$$

The verification of this last proposal can be done observing that:

The final temperature can be mesured by a thermometer, or calculated using the well-known equation:

$$
T_{f}=\frac{C_{1} T_{1}+C_{2} T_{2}}{C_{1}+C_{2}}
$$

where $C_{1}$ and $C_{2}$ are the specific heat capacities of part 1 and part 2. Knowing 
that the weight of 1 liter of water is $1 \mathrm{~kg}$ and admitting that $C p_{\text {water }}$ whose value is $4184 \mathrm{~J} \mathrm{~kg}^{-1} \mathrm{~K}^{-1}$, can be considered constant over the temperature interval taken into account, their values are:

$$
C_{1}=m_{1} c_{1}=1 \times 4184 \mathrm{~J} \cdot \mathrm{K}^{-1}, C_{2}=m_{2} c_{2}=3 \times 4184=12552 \mathrm{~J} \cdot \mathrm{K}^{-1}
$$

and the result obtained for $T_{f}$ is:

$$
T_{f}=313 \mathrm{~K}
$$

Then, introducing this value in the general equation:

$$
Q=\operatorname{MCp}\left(T_{f}-T_{i}\right)
$$

we get: $Q_{1}=125520 \mathrm{~J} \quad Q_{2}=-125520 \mathrm{~J}$ from which it can be seen that $Q_{s y s t}=Q_{1}+Q_{2}=0$, and therefore that Equation (21) $\left(d Q_{s y s t}=0\right)$ is effectively verified.

As concerns the change in entropy, the starting equation is:

$$
d S=\frac{d Q}{T}
$$

whose integration gives:

$$
\Delta S=\int_{T_{1}}^{T_{2}} \frac{d Q}{T}=M C_{P} \ln \frac{T_{f}}{T_{i}}
$$

Applying Equation (27) to part 1 and part 2 leads to

$$
\Delta S_{1}=421 \mathrm{~J} \cdot \mathrm{K}^{-1}, \Delta S_{2}=-395 \mathrm{~J} \cdot \mathrm{K}^{-1}
$$

Therefore, the change in entropy of the system is:

$$
\Delta S_{s y s t}=\Delta S_{1}+\Delta S_{2}=26 \mathrm{~J} \cdot \mathrm{K}^{-1}
$$

showing that the term $d S_{s y s t}$ obeys itself the relation:

$$
d S_{s y s t}>0
$$

In the same manner as we have seen, in Section 2, that the starting proposal $d U_{s y s t}=0$ (given by Equation (1)) raises a problem, we will see here that the starting proposal $d U_{s y s t}=0$ (isolated system) raises a similar problem. The reason is the following.

In thermodynamic textbooks, the concept of entropy is often introduced through an opposition between a reversible process and an irreversible process. It consists in saying that:

-When a system is concerned by a reversible process, its change in entropy is defined by the relation:

$$
d S=\frac{d Q}{T_{i}}
$$

where $T_{i}$ represents the internal temperature.

-When a system is concerned by an irreversible process, its change in entropy is defined by the relation:

$$
d S=\frac{d Q}{T_{e}}+d S_{i}
$$


where $T_{e}$ represents the external temperature and $d S_{i}$ an additional term, always positive, designated as the internal component of the entropy.

It is well known that, in practice, a process is always irreversible, but with a level of irreversibility more or less important. When the level of irreversibility is very low (i.e when the difference between $T_{e}$ and $T_{i}$ is very small), the term $d S_{i}$ tends towards zero and Equation (31) tends towards Equation (30).

In contrast with the term $d S_{p}$, the term $d S_{e}$, designated as the external component of the entropy, is defined as:

$$
d S_{e}=\frac{d Q}{T_{e}}
$$

Using these conventions, which were introduced in physical-chemistry by $\mathrm{J}$. W. Gibbs, the condensed expression of Equation (31) takes the form:

$$
d S=d S_{e}+d S_{i}
$$

The term $d Q$ having the same value in Equation (31) and Equation (32), (as will be verified hereafter) we can be tempted to think that, for a heat exchange, we have necessarily the relation:

$$
d Q_{i r r}=d Q_{r e v}
$$

Concerning this topic-which is the fundamental point of the present discussion-it can be noted that when a liter of water is heated from $283 \mathrm{~K}$ to $313 \mathrm{~K}$ (case examined above), the usual calculation of $\Delta Q$ is effectively independent of the level of irreversibility of the process. We get the same value $\Delta Q=125520 \mathrm{~J}$ when the water is directly put in contact with a thermostat at $313 \mathrm{~K}$, or heated progressively by contact with several heat sources at different temperatures, or by contact with another mass of water (such as the part 2 just considered) whose own temperature is decreasing from an initial value to the final value $313 \mathrm{~K}$.

Despite the fact that this interpretation is widely admitted and seems correct, the attention is called on the fact that Equation (31) is not an energy equation but an entropy equation. It can be converted into an energy equation multiplying each of its tems by $T_{e}$ and we get:

$$
T_{e} d S=d Q+T_{e} d S_{i}
$$

whose meaning is:

$$
d Q_{i r r}=d Q_{r e v}+d Q_{a d d}
$$

Combining the fact that $d S_{i}$ is positive with the fact that $T_{e}$ is an absolute temperature, the additional term designated $d Q_{a d d}$ appears to be necessarily positive.

From a comparison between the energy Equation (34) and Equation (35), and taking into account the information already given by the entropy Equation (30) and Equation (31), it appears that the terms $d Q_{\text {rev }}$ and $d Q_{i r r}$ can be directly defined as: 


$$
\begin{aligned}
& d Q_{\text {rev }}=T_{i} d S \\
& d Q_{i r r}=T_{e} d S
\end{aligned}
$$

The terms $T_{i}$ and $T_{e}$, being positive (absolutes temperatures), the sign of $d S$ is always the same as the sign of $d Q$. The consequence is that, for a given process (taking into account that $d S$ can be factorized, since $S$ is a state function) the difference between $d Q_{i r r}$ and $d Q_{\text {rer }}$ i.e.:

$$
d Q_{i r r}-d Q_{r e v}=d S\left(T_{e}-T_{i}\right)
$$

appears to be always positive, because we have $d S>0$ when $T_{e}>T_{i}$ and $d S<0$ when $T_{e}<T_{i}$.

As a consequence, the terms $d Q_{i r r}$ and $d Q_{r e v}$ are always linked together by the relation:

$$
d Q_{i r r}>d Q_{r e v}
$$

If we apply the integrated form of Equation (37) to both parts of the considered system, we must take into account the following peculiarities:

-For part 1: $T_{e}$ being the external temperature, it is the temperature of part 2 , but more exactly its average temperature that can be noted $T_{2}^{*}$. Its value is:

$$
T_{2}^{*}=\frac{\Delta Q_{2}}{\Delta S_{2}}=\frac{-125520}{-395}=318 \mathrm{~K}
$$

Therefore the value of the term $\Delta Q_{i r r .1}$ is itself:

$$
\Delta Q_{i r r .1}=\int_{283}^{313} T_{e} d S_{1}=T_{2}^{*} \int_{283}^{313} d S_{1}=318 \times 421=133878 \mathrm{~J}
$$

Having noted above that $\Delta Q_{\text {rev.1 }}=125520 \mathrm{~J}$, we get for $\Delta Q_{a d d .1}$ the value:

$$
\Delta Q_{\text {add.1 }}=8358 \mathrm{~J}
$$

-For part 2: A similar procedure leads to:

$$
\begin{gathered}
T_{1}^{*}=\frac{\Delta Q_{1}}{\Delta S_{1}}=\frac{125520}{421}=298 \mathrm{~K} \\
\Delta Q_{i r r .2}=\int_{323}^{313} T_{e} d S_{2}=T_{1}^{*} \int_{323}^{313} d S_{2}=298 \times(-395)=117710 \mathrm{~J} \\
\Delta Q_{a d d .2}=7810 \mathrm{~J}
\end{gathered}
$$

-For the system: Adding the values obtained above for the terms $\Delta Q_{\text {add.1 }}$ and $\Delta Q_{\text {add.2 }}$ the result obtained is:

$$
\Delta Q_{\text {add.syst }}=8358+7810=16168 \mathrm{~J}
$$

Obviously, such a result is not in adequacy with the conventional conception of thermodynamics, which lies on the idea that, in the case of an isolated system, the term called $d Q_{a d d}$ is necessarily nil, since no energy can be added or deducted.

Referring to this situation, some complementary observations are given below.

\section{The Link between Thermodynamics and Relativity}

It is well known that the difficulties encountered in learning thermodynamics 
are more conceptual than mathematical. Referring to the process just examined (heat exchange between two parts of an isolated system), the problem seems related to the fact that, if the entropy Equation (31) is totally accepted, its translation under the form of the energy Equation (34) and Equation (35) is generally absent from thermodynamic textbooks or courses.

The cause of this situation is probably the apparent impossibility to detect a physical symptom proving the reality of the energy symbolized by the designations $T_{e} d S_{i}$ or $d Q_{a d d^{*}}$

We have seen with Equation (39) that, for a given value of $d S$, a heat exchange is characterized by the relation $d Q_{i r r}>d Q_{r e v}$

In a similar way, it can be shown that, for a given value of $d V$, a work exchange is characterized by the relation:

$$
d W_{i r r}>d W_{r e v}
$$

This last proposal comes from the fact that the definitions of $d W_{i r r}$ and $d W_{r e v}$ are respectively:

$$
\begin{gathered}
d W_{i r r}=-P_{e} d V \\
d W_{r e v}=-P_{i} d V
\end{gathered}
$$

Therefore $d W_{i r r}-d W_{\text {rev }}=d V\left(P_{i}-P_{e}\right)$. Having $d V>0$ when $P_{i}>P_{e}$ and $d V<0$ when $P_{i}<P_{e}$ we are led to the conclusion given by Equation (40).

Remembering that for a system concerned by both an exchange of heat and an exchange of work, the change in internal energy $d U$ is defined as:

$$
d U=d Q+d W
$$

it appears that we have necessarily:

$$
d U_{i r r}>d U_{r e v}
$$

This proposal concerns indifferently a system exchanging energy with its surroundings or an isolated system which is the seat of an internal exchange of energy.

Taking into account that an isolated system is defined as having no exchange of energy with its surroundings, and that $d U_{\text {rev }}=0$, it seems that the inequality $d U_{i r r}>0$ must be systematically substituted to the equality $d U_{i r r}=0$ usually admitted.

Of course, we easily conceive that for the creators of the thermodynamic theory, it was impossible to imagine another condition than $d U=0$ for an isolated system. The situation is different today because, in the meantime, the concept of relativity has been introduced and particularly the Einstein mass-energy relation $E=m c^{2}$. Knowing that in this new prospect, a given amount of mass can be transformed in energy and conversely, the idea that an energy can be created (or destroyed) inside an isolated system becomes possible.

Under the light of this wider understanding, the suggestion has been advanced recently [9] [10] [11] that the doublet of Equation (34) and Equation (35) 
written above under the form:

$$
\begin{gathered}
T_{e} d S=d Q+T_{e} d S_{i} \\
d Q_{i r r}=d Q_{r e v}+d Q_{a d d}
\end{gathered}
$$

can be interpreted as meaning

$$
d Q_{i r r}=d Q_{r e v}-c^{2} d m
$$

In this last equation, the term $-c^{2} d m$ is the differential form of the Einstein relation, the minus sign recalling that an increase in energy is correlated with a decrease in mass, and conversely.

Adding to this conception the information $d U_{i r r}>d U_{r e v}$ given by Equation (44), the extended expression of Equation (45) takes the form:

$$
d U_{i r r}=d U_{r e v}-c^{2} d m
$$

From the practical point of view, it is important to observe that the term designated $d U_{\text {rev }}$ in Equation (46) has necessarily a zero value. The reason is that referring to Equation (41) and Equation (42), and having in mind the image of a gas contained in a cylinder with a piston, we easily conceive that in conditions of rigorous reversibility (i.e when $P_{e}=P_{i}$ ) we get $d V=0$ and thus $d W_{\text {rev }}=0$. The situation is similar for an exchange of heat because, referring to Equation (36) and Equation (37), the conditions of rigorous reversibility (i.e. $T_{e}=T_{i}$ ) lead to $d S=0$ and $d Q_{r e v}=0$. Adding both results, we get $d U_{r e v}=0$ and Equation (46) can be written under the reduced form:

$$
d U_{i r r}=-c^{2} d m
$$

From the theoretical point of view, the calculation of a reversible exchange in energy is nevertheless possible and is often done in themodynamics courses, particularly in the case of an ideal gas.

Concerning the nature of the energy created, and taking into account that it does not appear under the form of work or of heat, the hypothesis has been suggested that it can be a potential energy of gravitation [12]. This insight opens a possible relation between the decrease in mass of a planet and the progressive increase of its distance to other celestial bodies.

Correlatively, it has been suggested that for living matter, the term $d m$ of Equation (46) and Equation (47) could be positive, contrasting with inert matter where this term is interpreted as being negative [13].

\section{Conclusions}

The difficulties encountered in thermodynamics are more conceptual than mathematical. It seems that a possible answer lies in the idea that every irreversible process is an illustration of the Einstein mass-energy relation.

Equation (46) and Equation (47) are nothing but an attempt to insert this conception into the basic tools of the thermodynamic theory.

Among the other hypothesis explaining the behaviour of gaseous system, it is the one suggested by T. Yarman and A. L. Kholmetskii, based on quantum 
physics [14].

\section{Acknowledgements}

I would like to express my thanks to the readers who sent me positive comments about my previous papers on the subject.

\section{References}

[1] Tolman, R.C. (1928) On the Extension of Thermodynamics to General Relativity. Proceedings of the National Academy of Sciences of the United States of America, 14, 268-272. https://doi.org/10.1073/pnas.14.3.268

[2] https://en.wikipedia.org/wiki/Richard_C._Tolman

[3] Callen, H. and Horwitz, G. (1971) Relativistic Thermodynamics. American Journal of Physics, 39, 938. https://doi.org/10.1119/1.1986330

[4] Rengui, Y. (1996) The Logical Connection between Special Relativity and Thermodynamics. European Journal of Physics, 17, 265-267. https://doi.org/10.1088/0143-0807/17/5/003

[5] Haywards, S.A. (1999) Relativistic Thermodynamics. https://arxiv.org/pdf/gr-qc/9803007.pdf

[6] Farías, C.A., Moya, P.S. and Pinto, V.A. (2007) On the Relationship between Thermodynamics and Special Relativity. https://arxiv.org/abs/0712.3793

[7] Requardt, M. (2008) Thermodynamics Meets Special Relativity-or What Is Real in Physics? https://arxiv.org/abs/0801.2639

[8] Gupta, R.C., Gupta, R. and Gupta, S. (2010) Redefining Heat and Work in the Right Perspective of Second-Law-of-Thermodynamics. https://arxiv.org/abs/physics/0604174

[9] Tane, J.-L. (2000) Evidence for a Close Link between the Laws of Thermodynamics and the Einstein Mass-Energy Relation. Journal of Theoretics, 2, No. 3. http://www.journaloftheoretics.com/Articles/aArchive.htm

[10] Tane, J.-L. (2010) Unless Connected to Relativity, the First and Second Laws of Thermodynamics are Incompatible. https://arxiv.org/abs/0910.0781 and The General Science Journal http://gsjournal.net/.

[11] Tane, J.-L. (2014) An Extended Interpretation of the Concept of Entropy Opening a Link between Thermodynamics and Relativity. Natural Science, 6, 503-513. https://doi.org/10.4236/ns.2014.67049

[12] Tane, J.-L. (2008) Possible Impact in Astronomy of the Link between Thermodynamics and Relativity. The General Science Journal.

[13] Tane, J.-L. (2007) Thermodynamics and Relativity: A Basic Question about the Behavior of Living Matter. The General Science Journal.

[14] Yarman, T. and Kholmetskii, A.L. (2011) How Do Quantum Numbers Generally Vary in the Adiabatic Transformation of an Ideal Gas? Chinese Physics B, 20, No. 10. https://doi.org/10.1088/1674-1056/20/10/105101 
Submit or recommend next manuscript to SCIRP and we will provide best service for you:

Accepting pre-submission inquiries through Email, Facebook, LinkedIn, Twitter, etc. A wide selection of journals (inclusive of 9 subjects, more than 200 journals)

Providing 24-hour high-quality service

User-friendly online submission system

Fair and swift peer-review system

Efficient typesetting and proofreading procedure

Display of the result of downloads and visits, as well as the number of cited articles Maximum dissemination of your research work

Submit your manuscript at: http://papersubmission.scirp.org/

Or contact jamp@scirp.org 